\title{
Cancer bio-immunotherapy XVII annual NIBIT (Italian Network for Tumor Biotherapy) meeting, October 11-13 2019, Verona, Italy
}

\author{
Matteo Bellone ${ }^{1} \cdot$ Marco Bregni $^{2}$ - Vincenzo Bronte ${ }^{3}$. Stefano Ugel $^{3}$. Pier Francesco Ferrucci ${ }^{4} \cdot$ Massimo Di Nicola $^{5}$. \\ Paola Nisticò $^{6}$. Gaia Zuccolotto ${ }^{7}$. Antonio Rosato ${ }^{7,8} \cdot$ Vincenzo Russo $^{9} \cdot$ Antonio Sica $^{10,11} \cdot$ Mario P. Colombo $^{12}$ (1)
}

Received: 10 November 2020 / Accepted: 29 October 2021 / Published online: 9 November 2021

(c) The Author(s), under exclusive licence to Springer-Verlag GmbH Germany, part of Springer Nature 2021

Keywords Immunotherapy · Cancer vaccines · Checkpoint blockade agents · Adoptive immunotherapy · Targeted therapies $\cdot$ NIBIT

$\begin{array}{ll}\text { Abbreviations } \\ \text { AACR } & \text { American Association for Cancer Research } \\ \text { ACC } & \text { Alleanza Contro il Cancro } \\ \text { AIOM } & \text { Associazione Italiana di Oncologia Medica } \\ \text { ALL } & \text { Acute lymphoblastic leukemia } \\ \text { AML } & \text { Acute myeloid leukemia } \\ \text { CAF } & \text { Cancer-associated fibroblast } \\ \text { CAR } & \text { Chimeric antigen receptor } \\ \text { CCG } & \text { Clinical cancer genome } \\ \text { CICON } & \text { International Cancer Immunotherapy } \\ & \text { Conferences } \\ \text { CIMT } & \text { Association for Cancer Immunotherapy } \\ \text { CRI } & \text { Cancer Research Institute } \\ \text { CRPC } & \text { Castration-resistant prostate cancer } \\ \text { CTLA-4 } & \text { Cytotoxic T lymphocyte antigen-4 } \\ \text { DC } & \text { Dendritic cell } \\ \text { DLBCL } & \text { Diffuse large B-cell lymphoma }\end{array}$

Mario P. Colombo

mariopaolo.colombo@istitutotumori.mi.it

1 Unit of Cellular Immunology, I.R.C.C.S. Ospedale San Raffaele, Milan, Italy

2 Clinical Trial Center, I.R.C.C.S. Ospedale San Raffaele, Milan, Italy

3 Immunology Section, Department of Medicine, University and Hospital Trust of Verona, Verona, Italy

4 Unit of Tumor Biotherapy, Department of Experimental Oncology, I.R.C.C.S. European Institute of Oncology, Milan, Italy

5 Unit of Immunotherapy and Anticancer Innovative Therapeutics, Department of Medical Oncology, Fondazione I.R.C.C.S. Istituto Nazionale Tumori, Milan, Italy

6 Unit Tumor Immunology and Immunotherapy, I.R.C.C.S. Regina Elena National Cancer Institute, Rome, Italy

$\begin{array}{ll}\text { EATI } & \text { European Academy of Tumor Immunology } \\ \text { ENCI } & \text { European Network for Cancer Immunotherapy } \\ \text { ENO1 } & \alpha \text {-Enolase } \\ \text { HCC } & \text { Hepatocellular carcinoma } \\ \text { ICB } & \text { Immune checkpoint blockade } \\ \text { IFN- } \beta & \text { Interferon-b } \\ \text { IL-1R } & \text { Interleukin-1 receptor } \\ \text { IrAE } & \text { Immunotherapy-related adverse events } \\ \text { mAbs } & \text { Monoclonal antibodies } \\ \text { MDSC } & \text { Myeloid-derived suppressor cells } \\ \text { MM } & \text { Multiple myeloma } \\ \text { NIBIT } & \text { Italian Network for Tumor Biotherapy } \\ \text { NHL } & \text { Non-Hodgkin lymphomas } \\ \text { NSCLC } & \text { Non-small cell lung cancer } \\ \text { PDAC } & \text { Pancreatic ductal adenocarcinoma } \\ \text { PFS } & \text { Progression-free survival } \\ \text { RFS } & \text { Recurrence-free survival }\end{array}$

7 Department of Surgery, Oncology and Gastroenterology, University of Padova, Padova, Italy

8 Veneto Institute of Oncology IOV-IRCCS, Padova, Italy

9 Unit of Immuno-Biotherapy of Melanoma and Solid Tumors, IRCCS Ospedale San Raffaele, Milan, Italy

10 Molecular Immunology Lab, I.R.C.C.S. Humanitas Clinical and Research Center, Rozzano, MI, Italy

11 Department of Pharmaceutical Sciences, University of Piemonte Orientale "A. Avogadro", Novara, Italy

12 Molecular Immunology Unit, Department of Research, Fondazione I.R.C.C.S. Istituto Nazionale dei Tumori, Via Amadeo 42, 20068 Milan, Italy 


\begin{tabular}{|c|c|}
\hline RNS & Reactive nitrogen species \\
\hline SIC & Società Italiana di Cancerologia \\
\hline SImC & STS Immune Cluster \\
\hline SIICA & $\begin{array}{l}\text { Società Italiana di Immunologia ed Immunolo- } \\
\text { gia Clinica }\end{array}$ \\
\hline STS & Soft tissue sarcoma \\
\hline TLS & Tertiary lymphoid structures \\
\hline TIL & Tumor-infiltrating T lymphocyte \\
\hline TIM4 & $\begin{array}{l}\text { T-cell immunoglobulin and mucin domain } \\
\text { containing } 4\end{array}$ \\
\hline TME & Tumor microenvironment \\
\hline TSCM & T memory stem cells \\
\hline Tregs & Regulatory $\mathrm{T}$ cells \\
\hline TSLP & Thymic stromal lymphopoietin \\
\hline WGS & Whole genome sequencing \\
\hline Zeb1 & Zinc finger E-box-binding homeobox 1 \\
\hline
\end{tabular}

\section{Introduction}

Oncology embrace Immunology: the way forward captioned the XVII international meeting of the Italian Network for Tumor Biotherapy (NIBIT) held in Verona on October 11-13, 2019. In reporting below the excellence of the scientific program, we cannot refrain from recalling the beauty and the enjoyment of getting together for science education and promotion in a spirit of friendship and freedom. Participants also experienced the enchantment of the town celebrated by Shakespeare in Romeo and Juliet, which is also the capital of open-air lyric at the Romanic Arena, and the perfect place to taste the regional cuisine and the excellent Ripasso and Amarone wines.

The meeting also signed two relevant steps forward for the NIBIT. NIBIT launched a new partnership with Fondazione Pezcoller that sponsored the main keynote lecture. The President also announced the inception of the European Network for Cancer Immunotherapy (ENCI), of which NIBIT is founding member together with the Association for Cancer Immunotherapy (CIMT) and the European Academy of Tumor Immunology (EATI). ENCI has become the European partner of the American Association for Cancer Research (AACR) and the Cancer Research Institute (CRI) for the organization of the CICON International Cancer Immunotherapy Conferences. Jill O'Donnell-Tormey, chief executive officer and director of scientific affairs at CRI, graciously joined the meeting and delivered a talk on vision, persistence and impact of non-profit organizations in shaping the future of Immuno-Oncology. Themes of the meeting were: (a) the tumor microenvironment; (b) antigen presenting cells and active immunotherapy; (c) adoptive immunotherapy; (d) efficacy of immune checkpoint inhibitors in sensitive and more resistant tumors; and e) clinical and research highlights around NIBIT. The meeting was organized in the following sessions.

\section{Session 1:Tumor microenvironment}

As appreciation for the collaborative efforts between the NIBIT and other Italian scientific societies, Session 1 was co-chaired by the NIBIT Past President Vincenzo Russo (Milan, Italy) and Marco Cassatella (Verona, Italy), vice president of the Società Italiana di Immunologia and Immunologia Clinica (SIICA). The tumor microenvironment (TME) has a central role in restraining tumor growth, and a deep understanding of the interplay between cancer cells and their local environment is relevant to designing immunotherapies that fit each tumor best. Session 1 was dedicated to recent advancements in the comprehension of the TME. Wolf-Herman Fridman (Paris, France) discussed on the relevance of the tertiary lymphoid structures (TLS) as potential predictive biomarkers in soft tissue sarcomas (STSs). Fridman's group focused on leiomyosarcoma, dedifferentiated liposarcoma and undifferentiated pleomorphic sarcoma and classified them on the basis of the abundance of different immune and stromal cells using a transcriptomicbased deconvolution method (MCP-Counter immune cell signatures). They identified 5 STS Immune Clusters (SImC), named A, B, C, D and E, endowed with different features. The SImC E had the highest lymphocytic infiltration, immune checkpoint expression and were characterized by TLSs. Patients whose tumors were classified SImC E had a better overall survival with the $\mathrm{B}$ cell signature behaving as a better predictor than cytotoxic signature. Patients within the SImC E cluster also experienced the highest response to PD-1 blockade [1]. Fridman also illustrated the importance of the complement pathway in clear cell renal carcinoma. He showed that high density of $\mathrm{C} 1 \mathrm{q}, \mathrm{C} 3$ and $\mathrm{C} 4$ producing cells and of $\mathrm{C} 4$ deposits correlated with poor prognosis in clear cell renal carcinoma [2]. Sophie Lucas (Brussels, Belgium) showed that human regulatory $\mathrm{T}$ cells (Tregs) mediate immunosuppression by activating latent cell surface TGF- $\beta 1$ via a mechanism that requires GARP and the integrin $\alpha \mathrm{V} \beta 8$. She also showed that blocking with anti-GARP/TGF- $\beta 1$ monoclonal antibodies (mAbs) inhibited active TGF- $\beta 1$ production and immunosuppression by human Tregs [3]. Of note, by immunofluorescence analyses she displayed human melanoma metastases infiltrated by GARP+Tregs, which showed high levels of TGF- $\beta$ signaling when analyzed by RNA-seq. Finally, she showed that anti-GARP/TGF- $\beta 1 \mathrm{mAbs}$ combined with anti-PD-1 mAbs in tumor-bearing mice had a superior antitumor effect when compared to single therapies [4]. Triantafyllos Chavakis (Dresden, Germany) discussed the concept of trained innate immunity and showed results highlighting the fact that trained immunity acts via 
modulation of hematopoietic stem and progenitor cells. Indeed, mice administered with the prototypical trainedimmunity-inducing agonist $\beta$-glucan underwent the expansion of myeloid lineage progenitors, associated with elevated signaling by IL-1 $\beta$ and granulocyte-macrophage colonystimulating factor. The expansion of myeloid progenitors resulted in a beneficial response to secondary LPS challenge, which protected mice from chemotherapy-induced myelosuppression. Pre-treatment of mice with $\beta$-glucan resulted in diminished tumor growth. This effect was associated with granulopoiesis and neutrophil reprogramming toward an anti-tumor phenotype. The adoptive transfer of neutrophils from $\beta$-glucan-trained mice to naive recipients suppressed tumor growth in a ROS-dependent manner [5]. Catherine Sautès-Fridman (Paris, France) discussed the role of TLSs in hepatic tumors. She showed data highlighting early TLS formation and immune activation occurring in hepatocellular carcinoma (HCC). The presence of intratumoral TLS correlated with decreased frequency of early tumor recurrence and better prognosis in HCC [6, 7]. Silvia Piconese (Rome, Italy) described 2 subsets of Tregs in preclinical tumor models, i.e. Treg cells expressing TNFR2 and a subset producing TNF. She showed that during in vitro activation, TNF produced by Tregs promotes TNFR 2 upregulation by an autocrine mechanism. This effect leads to a TNF-TNFR2 signaling amplification in Tregs, ultimately supporting Treg proliferation. This mechanism is supposed to further sustain the pro-tumoral role of TNF at the tumor site. Francesca Di Modugno (Rome, Italy) reported on isoforms of hMENA, a member of the Ena/VASP protein family that regulates actin dynamics, integrin-mediated signaling, cell-adhesion and cell motility. hMENA inversely regulates the expression of LT $\beta$ R in tumor cells and CAFs, thus influencing the TLS localization and the prognosis of early node-negative non-small cell lung cancer (NSCLC) patients [8]. Moreover, she reported that the definition of tumor and stromal hMENA isoform expression pattern defines subclasses of tumor immune microenvironment.

\section{Session 2: Antigen presenting cells and active immunotherapy}

Session 2 was co-chaired by Paola Nisticò (Rome, Italy) and Massimo Di Nicola (Milan, Italy), and was mainly focused on the role of antigen presenting cells as source of promising powerful approaches in cancer treatment. Carl Figdor (Nijmegen, The Netherlands) highlighted the efficacy of dendritic cell (DC)-based cancer vaccines as a more tolerable strategy to expand the tumor reactive $\mathrm{T}$ cell pool. In particular, the author discussed the need to target DCs in vivo through the engineering of $3 \mathrm{D}$ biomaterial-based scaffolds. These structures represent synthetic immune niches, able to mimic natural lymph nodes and to provide novel sites for immune-cell interaction. Synthetic immune niches can be exploited to modulate the immune response in alternative locations, allowing incoming leukocytes to interact with molecular signals coming from the scaffolds. These approaches enable local immunomodulation and address the limitations of current immunotherapeutic strategies. Nicholas McGranahan (London, United Kingdom) revealed the heterogeneous landscape of NSCLC and showed an impressive analysis on the correlation between genomically similar tumor regions and their immune microenvironment, indicating the role of the immune system in sculpting the evolution of the tumor genome. He proposed the integration of genomic, transcriptomic, epigenomic results with pathologic data to predict immune evasion capacity and disease-free survival in early NSCLC patients. Patients with either a high clonal neoantigen load or low immune-evasion capacity exhibited significantly increased disease-free survival [9]. Federica Benvenuti (Trieste, Italy) indicated T-cell immunoglobulin and mucin domain containing 4 (TIM4), a receptor for phosphatidylserine implicated in uptake of dying cells (efferocytosis) by macrophages, as involved in tissue surveillance in the lung. She revealed TIM4 down-regulation as a mechanism of tumor immune evasion and demonstrated as TIM4 loss in lung DCs1 impairs immune-mediated tumor control. Mattia (Bologna, Italy) highlighted that colorectal cancer patients resistant to cetuximab, an inhibitor of epidermal growth factor receptor, have an increased expression of inflammatory cytokines and high level of interleukin-1 receptor (IL-1R), which is predictive of shorter survival. He proposed the combination of IL1R inhibition with cetuximab to overcome resistance. Gaetano Finocchiaro (Milan, Italy) explored the role of DC-based immunotherapy in recurrent glioblastoma multiforme. The author reported data from two studies, the DENDR2 and its variant (V)-DENDR2. The difference between the two protocols was the presence of a pre-conditioning step in which, patients treated with DCs and temozolomide, were also injected locally with a tolerated dose of tetanus toxoid. Data demonstrated a role for the pre-conditioning step in increasing the $\mathrm{T}$ helper immune response, encouraging larger studies aimed at combining DC vaccination with standard therapies after pre-conditioning steps [10].

In the section dedicated to selected abstracts, Barbara Bassani (Milan, Italy) discussed the role of Zinc finger E-box-binding homeobox 1 (Zeb1) in acute myeloid leukemia (AML). In particular, the author analyzed Zeb1 expression in cytogenetically negative AML, reporting that murine cells overexpressing Zeb1 showed higher levels of PD-L1, IL-2 and lower levels of CD40, IL-6, TNF $\alpha$ and IL-10 than WEHI-3B cells. Zeb1 silencing induced the downregulation of immune-regulatory genes including PD-L1, Arg1, Tgf $\beta$ and OX40L and of pro-metastatic genes, such Mmp9. The 
relevance of Zeb1 in leukemia aggressiveness was confirmed also in humans. Indeed, a positive correlation between Zeb1 and PD-L1, and an association with a poor outcome was observed in AML patients stratified for Zeb1 expression.

\section{Session 3: Adoptive immunotherapy}

Session 3 was a joint session between the NIBIT represented by Antonio Rosato (Padova, Italy) and Ruggero De Maria (Rome, Italy) president of the Alleanza Contro il Cancro (ACC) association. Session 3 focused on adoptive immunotherapy, especially employing genetically modified $\mathrm{T}$ lymphocytes, which represent one of the most innovative approaches for cancer treatment. Fabio Ciceri (Milan, Italy) opened the session with a comprehensive overview about the state-of-the-art of chimeric antigen receptor (CAR)-transduced $\mathrm{T}$ cells in hematological malignancies. He focused on some aspects related to CAR-T toxicities like the cytokine release syndrome CRS [11], the CAR-T-related encephalopathy syndrome, on off target tumor and off target toxicities. Ciceri also presented clinical data about current ongoing CAR-T trials in acute lymphoblastic leukemia (ALL), non-Hodgkin lymphomas (NHL) and multiple myeloma (MM). Finally, he briefly discussed about the evolution of CAR-T and non-viral approaches for CAR expression, as the sleeping beauty transposon system that is employed in an ongoing clinical trial for adult and pediatric ALL and sponsored by the Tettamanti Foundation (Monza, Italy), where the adoptively transferred effectors are represented by cytokine-induced killer cells expressing an anti-CD19 CAR (CARCIK-CD19; NCT03389035). Catia Traversari (Milan, Italy), Research Director at the MolMed biotech company, discussed about all the preclinical research activities involving a CAR directed against the v6 variant of the CD44 adhesion molecule (CD44v6), for the potential treatment of hematopoietic and solid cancers. Targeting the CD44v6 antigen with CD44v6.CAR-T has been reported to control the neoplastic growth in a high tumor burden mouse model of AML and multiple myeloma (MM), with limited off-target toxicities. Such results constitute the premises for a multicenter, first-in-man phase I/IIa clinical trial that is conducted within the EU-funded EURE-CART project and aims at evaluating the safety and efficacy of CD44v6.CAR-T cells co-expressing the HSV-TK suicide gene in relapsed/refractory AML and MM (NCT04097301). CD44v6.CAR-T cells are also under investigation for the therapeutic treatment of several other neoplastic conditions such as ovarian cancer, lung adenocarcinoma, sarcoma and melanoma. Collectively, results obtained both in xenogeneic and syngeneic models support the concept the CD44v6.CAR-T restrain tumor growth, inhibit experimental metastasis, improve survival and also synergize with anti-CTLA-4 and anti-PD-1 immune checkpoint blockade therapy [12]. Luca Gattinoni (Regensburg, Germany) discussed the use of CD8+ T memory stem cells (TSCM) transduced with CD19-specific CAR for the treatment of human B-cell malignancies [13]. He illustrated the feasibility of a robust clinical-grade platform for the generation of CD19-redirected CAR-modified TSCM endowed with high antitumor activity. He also provided evidence that these cells are therapeutically superior to CD8+ T cell products generated with clinical protocols currently under investigation. CD19CAR-modified CD8+ TSCM exhibit enhanced metabolic fitness and mediate robust, long-lasting antitumor responses in ALL xenograft models, and appear also very promising in early clinical translation. In the selected abstracts section, Francesco De Sanctis (Verona, Italy) showed how the nitrosative stress modulation of pancreatic TME can favor the efficacy of adoptively transferred telomerase (TERT)-specific cytotoxic T lymphocytes. TERT deregulation is found in several solid tumors, and treatments targeting telomerase might be useful also for the therapy of pancreatic ductal adenocarcinoma (PDAC). PDAC progression is also accompanied by the establishment of a chemical barrier dependent on reactive nitrogen species (RNS), which impairs T cell tumor infiltration. De Sanctis demonstrated that pharmacological treatment reducing RNS production favors the efficacy of adoptively transferred $\mathrm{T}$ cells transduced with a HLA-A2-restricted T cell receptor (TCR) recognizing human TERT with high avidity. Gaia Zuccolotto (Padova, Italy) reported a valid and cost-effective alternative to CAR-modified T cells. This novel therapeutic approach against prostate cancer is based on the engineering of immortalized NK-92 cells with a CAR recognizing the human prostate-specific membrane antigen, which is overexpressed in prostatic neoplastic cells. NK-92/CAR cells not only showed a potent antitumor activity in vitro but the adoptive transfer of irradiated NK-92/CAR cells in prostate cancer-bearing mice restrained tumor growth and improved survival [14]. Gaia Zuccolotto was awarded with the NIBIT Best Basic Paper Award and Matteo Catucci (Milan, Italy) with the NIBIT Best Translational Paper Award, both sponsored by the Fondazione Grazia Focacci.

\section{Session 4: Modulating immunotherapy in solid tumors}

Immune checkpoint blockade (ICB) has revolutionized cancer immunotherapy with strategies that can selectively target specific or innate immunity. Session 4 was dedicated to ICB and co-chaired by Antonio Sica (Milan, Italy) and Gabriella Sozzi (Milan, Italy), president of the Società Italiana di Cancerologia (SIC). Within this perspective, Ranjana Advani (Stanford, USA) presented a phase 1b study involving patients with relapsed or refractory non-Hodgkin's 
lymphoma, treated with a combination of $5 \mathrm{~F} 9$, an antibody against the macrophage "do not eat me" signal CD47, and rituximab (anti-CD20; ref. [15]). The study enrolled 22 patients (15 with diffuse large B-cell lymphoma and 7 with follicular lymphoma). Principal adverse events were of grade 1 or 2 . Fifty $\%$ of the treated patients had an objective response, and $36 \%$ experienced a complete response. Overall, the results indicated the combination of the checkpoint inhibitor 5F9 with rituximab could be a promising therapy in patients with aggressive and indolent lymphoma, with no clinically significant adverse events. Sandra Demaria (New York, USA) discussed the use of radiation therapy to enhance responses to immune checkpoint inhibitors, focusing on anti-CTLA-4. She summarized the published preclinical data showing that accumulation of cytosolic DNA that follows radiation-induced DNA damage induces cancer cells to produce IFN- $\beta$ via the cGAS/STING pathway [16]. Irradiated cancer cells also produce exosomes that carry the tumor-derived DNA to DCs, leading to STING-dependent IFN- $\beta$ production by DCs [17]. Type I IFN is required to enhance tumor infiltration by DCs and promote cross-presentation of tumor antigens by DCs to CD8 T cells. Activation of tumor-specific CD8 T cells that mediate regression of the irradiated tumor as well as a non-irradiated synchronous tumor (abscopal effect) is seen only when radiation is combined with CTLA-4 blockade, whereas each treatment alone was ineffective, demonstrating a therapeutic synergy of radiation and CTLA4 blockade. She also reported on a clinical trial in metastatic chemo-refractory non-small cell lung cancer (NSCLC) patient. Focal radiation therapy delivered to a single metastasis with anti-CTLA4 antibody ipilimumab resulted in objective systemic responses in $18 \%$ of the intentto-treat population, and $31 \%$ had disease control. Increased serum IFN- $\beta$ after radiation and early dynamic changes of circulating $\mathrm{T}$ cell clones were the strongest response predictors, confirming preclinical mechanistic data [18]. She concluded showing unpublished data providing new insights into the synergy between radiation and anti-CTLA-4 observed in both mice and patients. Marina Garassino (Milan, Italy) focused her talk on recent advancements in NSCLC treatment by immunotherapy. Despite the encouraging results obtained with pembrolizumab in patients expressing PD-L1 at high levels, single agent immunotherapy did not demonstrate to be superior to the standard chemotherapy approximately in $70 \%$ of patients. Because chemotherapy induces immunogenic cell death and kill some myeloid suppressor component, resulting in activation of $\mathrm{T}$ cells and reduction of immunosuppression, several chemotherapeutic agents have been tested in combination with ICB. Based on this rationale, phase III clinical trials of combination between chemotherapy and anti-PD-L1 in metastatic NSCLC patients have been conducted with excellent results [19, 20]. Discussion is still ongoing on the significance of PD-L1 expression for patients' stratification and selection. Tumor mutational burden assessed on both tumor tissue and blood plays a role in the selection of single agent immunotherapy, but not in combinations with chemotherapy. Several other agents have been tested in combination with the first generation of immunotherapy, with poor results. This is potentially due to the fact that the complexity of the microenvironment is not yet well understood. Some results have been observed with combinations of anti PD-1, anti-angiogenic and antiTIGIT. The future challenge of lung cancer research will be the selection of suitable treatments and the discovery of new agents capable of overcoming the frequent loss of HLA. Ignacio Melero (Pamplona, Spain) discussed the multifaced aspects of cytokines in cancer. Indeed, the cytokine family possesses immunostimulatory or immunosuppressive capacities overall, and their implementation in cancer treatment deserves particular attention. For example, the adoptive transfer of engineered $\mathrm{T}$ cells retrovirally transferred with the IL-12 gene elicited strong antitumor efficacy, but with unacceptable severe side effects. Conversely, tumor-specific CD8+ T cells engineered to transiently express IL-12 and injected intratumorally led to complete tumor rejections, with low toxicity [21]. Emerging evidence further indicates that the prophylactic blockade of TNF before treatment with anti-CTLA-4 and anti-PD-1 prevents autoimmune adverse events, and also enhances to some extent the anti-tumor efficacy of the combined treatment in mouse models [22]. Melero also reported that elevated serum IL-8 is associated with enhanced intratumor neutrophils and reduced clinical benefit of immune-checkpoint inhibitors [23]. Marino Kallikourdis (Milan, Italy) intrigued the audience with a talk focused on tissue-dependent and context-dependent roles of type 2-polarized responses during carcinogenesis and tumor growth. In particular, Th-2-polarized T cells and macrophages favor fibrosis, generating a wall around the tumor mass, thus hindering cytotoxic T cell infiltration. Indeed, in the absence of $\mathrm{T}$ cells, mice show reduced scar formation [24]. These findings are relevant for optimizing the timing of cancer immunotherapy in patients.

\section{Session 5: Modulating immunotherapy in melanoma and other immune sensitive tumors}

Matteo Bellone (Milan, Italy) and Marco Bregni (Milan, Italy) co-chaired Session 5, which focused on ICB in melanoma and other immune sensitive neoplasms. Whereas the gut microbiota has been shown to impact cancer progression and clinical response to ICB, less investigated is its role in modulating the response to monoclonal antibodies specific for tumor associate antigens. Elda Tagliabue (Milan, Italy) opened Session 5 reporting intriguing data on the correlation 
between the response of breast cancer patients to treatment with trastuzumab, a recombinant humanized monoclonal antibody that binds to the HER2 extracellular domain, and the gut microbiota. In particular, non-responding patients were characterized by a unique bacterial community in their intestinal microbiota. They are investigating in relevant mouse models the impact of those commensals on the immune response triggered by trastuzumab treatment. It was discussed on the potential pathogenic role of microbiota-propelled Thelper 17 cells in lung cancer and other tumors [25]. The role of the lung microbiota as biomarker for lung cancer, as well as novel target for therapeutic interventions was also touched upon in the discussion [26]. Christian Blank (Amsterdam, The Netherlands) focused his talk on neoadjuvant immunotherapy and showed that neoadjuvant treatment with the combination of nivolumab and ipilimumab in stage III melanoma patients expanded more and broader tumor resident $\mathrm{T}$ cell clones than adjuvant treatment [27]. A key to success is the identification of the optimal combination dosing schedule to reduce the risk of immunotherapy-related adverse events (IrAE) without reducing clinical responses [28]. Interim analysis of the OpACIN-neo study showed that at 18 months the recurrence-free survival (RFS) of stage III melanoma patients was similar in the three arms under investigation, and the pathologic response remained an excellent surrogate marker for RFS. Thus, reverse translation, in which data from biopsies and surgical specimens are used to select therapies in an individualized manner, is an optimal tool to improve patient outcomes [29]. Pier Francesco Ferrucci (Milan, Italy) discussed the rationale of combining ICB and targeted therapy, focusing on the KN022 part 3 , a randomized trial that combines dabrafenib, trametinib and pembrolizumab versus target therapy alone. He also reported on two ongoing clinical trials: the IMspire 150 (Vemurafenib-Cobimetinib and Atezolizumab) and COMBII (Dabrafenib-Trametinib and Spartalizumab). Interestingly, in BRAF-mutated melanoma patients, the triplets treatment conferred numerically longer progression-free survival (PFS) and duration of response compared to treatment with dabrafenib, trametinib and placebo. The triplet therapy was also associated with a higher rate of grade 3/4 adverse events [30]. Luna Minute (Navarra, Spain) reported exciting data on the induction of immunogenic cell death caused by cytotoxic $\mathrm{T}$ lymphocytes and natural killer cells, which also induced epitope spreading, thus increasing the anti-tumor activity of the immune response [31]. The presentation by Maria Teresa Majorini (Milan, Italy) was focused on the role that mast cells may have in breast cancer. Starting from the observation that mast cells are more infiltrated in luminal hormonepositive breast cancer in humans, she found that mast cells activated a luminal program in mouse breast cancer cells, whereas the lack of mast cells favored the development of tumors with a basal phenotype. By activating estrogen receptor-mediated pro-survival signaling, mast cells also appeared to promote resistance to trastuzumab therapy [32]. Thus, mast cells may directly affect the phenotype of cancer cells, and their susceptibility to cancer therapies. Marta Truffi (Pavia, Italy) closed the session presenting a new approach to increase sensitivity of breast cancer cells to trastuzumab. She showed that in vitro, the half chain fragment of trastuzumab conjugated to iron oxide colloidal nanoparticles efficiently bound to HER2+ breast cancer cells, and was internalized, eventually reducing cell proliferation and promoting antibody-dependent cellular cytotoxicity [33].

At the end of Session 5, Enzo Galligioni the President of the Pezcoller Foundation, based in Trento (Italy), briefly summarized the many activities promoted by the Foundation in favor of science, which also include sponsoring prestigious international prizes and lectures. For the Pezcoller premier lecture, the audience appreciated Padmanee Sharma (Houston, TX, USA), who spoke on a discovery-driven approach in which hypothesis testing begins in more realistic clinical settings among patients participating in clinical trials. The contemporary existence of multiple checkpoints and their dynamic expression need their evaluation pre- and during treatment to understand resistance and guide new treatment also in search of suitable biomarkers. The specificity of organ microenvironment is also an important variable affecting the immune response against the invading tumors. The in-depth discussion that followed was enriched from the presence in the audience of Nobel Laureate Jim Allison, also a previous recipient of the Pezcoller/AACR Prize. He honored the NIBT in accepting the NIBIT Carrier Award.

\section{Session 6: Focus on pancreatic adenocarcinoma and other immune resistant tumors}

Vincenzo Bronte (Verona, Italy) and Michele Milella (Verona, Italy) led the discussion in Session 6, which investigated pancreatic cancer and other immune resistant tumors. Advances in sequencing technology have provided a near-complete glossary of genomic cancer drivers. The ability to target pharmacologically several of these diverse oncogenic molecules has raised the expectation that a precision medicine approach could improve patient outcomes. However, the implementation of genomics in therapeutic development and healthcare poses the need for an effective, yet flexible, genomic platform that operates in realworld setting to capture clinically relevant information in solid tumors with multiplex testing efficiency to reduce costs. Aldo Scarpa (Verona, Italy), discussed the development of clinical cancer genome (CCG) assay, a multiplex assay specifically designed to overcome the technical limitations of whole genome sequencing (WGS) in fixed and low 
cellularity samples, whilst still capturing an equivalent range of genomic features. Differently from many available cancer panels, the CCG panel covers a more comprehensive list of genomic events including structural variations, microsatellite instability, genome-wide signatures, which have been demonstrated to be informative for cancer diagnosis and therapeutic response [34]. Lucia De Monte (Milan, Italy), delineated the immune composition of pancreatic ductal adenocarcinoma (PDAC) framework. PDAC microenvironment is characterized by a marked Th2 inflammation, which is promoted by thymic stromal lymphopoietin (TSLP) secreted by cancer-associated fibroblasts (CAFs). Indeed, TSLP induces the activation and maturation of Th2 polarizing DCs and promotes macrophage polarization towards M2 cells. TSLP release mainly depends on the ability of cancer cells to release the inflammasome adaptor, which induces IL-1 $\beta$ secretion by macrophages [35]. Indeed, the IL-1 receptor antagonist (Anakira)-based treatment can control TSLP release by CAFs, both in vitro and in vivo [36]. Immunomodulatory agents able to reprogram this complex interplay between cancer cells and tumor-infiltrating leukocytes might open new therapeutic frontiers on PDAC. Andrea Alimonti (Bellinzona, Switzerland) discussed the impact of myeloid-derived suppressor cells (MDSCs) on the development of resistance to androgen-deprivation therapy, a condition leading to castration-resistant prostate cancer (CRPC) development. He highlighted the impact of IL-23 produced by MDSCs as key players in CRPC. Indeed, IL-23 favors the activation of the androgen receptor pathway in prostate cancer cells, promoting cell survival and proliferation. These extrinsic factors of tumor-associated immune resistance were abrogated by antibody-mediated inactivation of IL-23. Indeed, anti-IL23 therapy restored sensitivity to androgen-deprivation therapy in tumor-bearing mice [37]. Therefore, altering the composition of soluble factors in the tumor microenvironment might be exploited as valid strategies to overcame tumor resistance to conventional treatments.

Paola Cappello (Torino, Italy) illustrated the ability of anti-tumor DNA vaccine targeting $\alpha$-enolase (ENO1) antigen to control tumor growth in PDAC-bearing mice by promoting the expansion of $\mathrm{T}$ cells that specifically recognize ENO1. Interestingly, this therapeutic effect could be enhanced by IL-17 abrogation. Indeed, the genetic or pharmacological targeting of IL-17 strongly improved the expansion of $\mathrm{T}$ cells with an effector/memory phenotype as well as a contraction in $\mathrm{T}$ regulatory cells. These data argue that immune response to PDAC antigens might be overcome by manipulating the cytokine milieu in tumorbearing hosts. Claudio Bassi (Verona Italy) delivered the final talk of this session about the potential revolution in the treatment of PDAC patients. Indeed, chemotherapy regimens, such as FOLFIRINOX and Gemcitabine plus
Nab-paclitaxel, exhibited favorable results in advanced settings, allowing an increased surgical opportunity for patients affected by locally advanced and even metastatic cancers. Today, treatment allocation of PDAC patient is currently based on clinical, biochemical, and radiologic features but, to reach a personalized treatment, it is mandatory to identify new tools to predict tumor response and develop a more effective patient treatment. Therefore, PDAC management is now moving from a radiology-driven to a biology-driven decision-making process.

\section{Session 7: Clinical and research highlights around NIBIT}

The seventh and last session was co-chaired by Mario P. Colombo (Milan, Italy), NIBIT President, and Stefania Gori (Milan, Italy) as President of the Associazione Italiana di Oncologia Medica (AIOM). Presentations were focused on reviewing new data on immuno-oncology applications with a more clinical perspective. Roberto Bordonaro (Catania, Italy), reported on currently ongoing trials with ICB that are revolutionizing most of the approaches in different diseases, becoming a standard treatment in melanoma, lung cancer, renal cancer and even head/neck cancers. ICB has been combined with chemotherapies, and molecular or biological therapies aiming at enhancing patient's benefit from the synergy of drugs addressing different targets. Massimo Guidoboni (Meldola, Italy), reviewed the recent history of DC-based vaccines, which induce effective anti-tumor immunity, although often without translation into durable clinical responses. To better understand the mechanisms underlying the immuno-editing processes occurring upon DC vaccination what happens in the TME should still be properly elucidated. In particular, quantitative and qualitative changes in tumor-infiltrating T lymphocytes (TILs) has been shown to exert an important role in sustaining or de novo inducing a $\mathrm{T}$ cell inflamed TME [38]. The strength of intratumoral $\mathrm{T}$ cell activation detected in post-DC therapy lesions is also lessened by an occurring phenomenon of adaptive immune resistance with concomitant PD-L1 upregulation. Combining DC vaccination with ICB could be an option to ameliorate clinical results.

The field of cancer vaccine development for liver cancer has been discussed by Luigi Buonaguro (Naples, Italy), who explained how an effective immunotherapy in $\mathrm{HCC}$ requires the combination of an immune activator with immune modulators, aiming at compensating the strong liver immune suppressive TME. Promising strategies are represented by the combination of a cancer vaccine with immunomodulatory drugs, such as chemotherapy and ICB in order to overcome resistance. Preclinical data demonstrated that combinatorial strategies are effective in potentiating the effector functions 
of activated T cells and to decrease the density of immunosuppressive cells in the TME. Recent studies also confirmed clinical effectiveness of ICB in liver cancer, supporting the rationale of combining ICB with cancer vaccines. Among the ongoing clinical trials, the Hepavac-101 (NCT03203005) aims to identify more specific target antigens in order to improve cancer vaccination efficacy. Serena Di Cosimo (Milan, Italy), showed how CDK4/6 inhibitors (Palbociclib, Ribociclib, Abemaciclib) combined with endocrine therapy have improved response rates and survival in advanced hormone receptor-positive, HER2-negative, metastatic breast cancer. Their mechanism of action, by selective CDK4 and CDK6 inactivation, ends up in in restoring the growth-suppressive properties of the retinoblastoma protein and blocking the cell cycle. Recent work has also revealed that the antitumor activity of CDK4/6 inhibitors rely on unleashing the immune system to attack cancer cells.

Sriram Sathy (Cambridge, USA) presented preclinical data on exoASO-STAT6 and exoASO-C/EBP $\beta$ programs, utilizing the company's proprietary engEx ${ }^{\mathrm{TM}}$ Platform to engineer exosomes exogenously loaded with an antisense oligonucleotide (ASO). These exosomes are designed to selectively reprogram M2 immunosuppressive macrophages to an M1 pro-inflammatory phenotype by targeting and decreasing the expression of the immunosuppressive transcription factors STAT6 and C/EBP $\beta$. Both platforms showed significant efficacy when administered intravenously in an aggressive orthotopic model of HCC. Interestingly, this platform allows designing exosomes with precisely engineered properties, which incorporate various types of biologically active molecules and direct them to specific cell types and tissues. Emilia Cocorocchio (Milan, Italy) focused her talk on immunotherapy advances in melanoma. She reported data from ongoing clinical trials combining antiPD- 1 or anti-CTLA-4 antibodies to locoregional treatments, such as T-VEC (Amgen) or IMO-2125 (IDERA Pharmaceuticals). T-VEC (Talimogene Laherparepvec) is a genetically engineered oncolytic herpes virus carrying the human GMCSF gene, which is directly injected in a cutaneous or subcutaneous lesion. T-VEC showed activity in monotherapy, and when combined with anti-PD-1 appeared to revert acquired resistance. IMO-2125 is an investigational intratumoral tolllike receptor 9 agonist that modulates the TME to enhance anti-tumor immunity. A phase 3 trial is ongoing to compare its efficacy when combined to anti-CTLA4 in patient progressing to mono-immunotherapy (NCT03445533). Both these associations have a strong biological rationale, since targeting different molecules in different sites of the disease could end up in enhancing the host immune response. Martina Angi (Milan, Italy) underscored that uveal melanoma is an orphan disease with relevant clinical unmet needs. The biology and genetics of uveal melanoma diverge from that of skin melanoma [39], making the former less sensitive to
ICB. Unfortunately, approximately $50 \%$ of patients affected by uveal melanoma progress to liver metastasis. Tebentafusp is a novel immunotherapy drug developed by Immunocore on a very innovative platform called immune-mobilizing monoclonal $\mathrm{T}$ cell receptor against cancer (ImmTAC). ImmTAC consists of a soluble, affinity enhanced TCR fused to an anti-CD3 single-chain variable fragment. It is engineered to specifically target gp100, redirecting T lymphocytes to melanoma cells. Pivotal clinical trials are currently underway in HLA-A2 patients [40].

\section{Conclusions}

The XVII NIBIT meeting unfolded in the enchanted fall of the charming Verona and its surroundings. Basic, translational and clinical scientists confronted each other in a tightscheduled program of lectures, invited talks and selected abstract presentations. Attendees were updated on the most recent discoveries and results from both basic investigations and clinical trials, and they benefited from discussing with leaders in cancer immunology and immunotherapy. They strengthened scientific collaborations and built the basis for new ones. Young scientist had the unique opportunity to present their findings in a very informal environment, test their skills and confront with opinion leaders.

In conclusion, the meeting established the central role of the immune component of TME in conditioning the efficacy of immunotherapies, including immune-checkpoint inhibitors, CAR-T, and DC-based vaccination even when they were combined with radio- and chemotherapies. As such, the meeting was a success, and reinforced the NIBIT mission of putting together clinicians and basic scientists to distill new idea and concepts of translational applicability.

The meeting was adjourned with the promise to get together again in 2020 for the "XVIII NIBIT Meeting".

Acknowledgements This meeting was organized in collaboration with the NIBIT Board of Directors.

Author contributions All authors contributed to writing the manuscript. MB collected and assembled contributions from all authors. All authors revised and approved the final version of the manuscript.

Funding This meeting was supported in part by unrestricted grants from Bristol-Meyers Squibb, Novartis, Roche, Akoya, AstraZeneca, Codiak, Gilead, Idera, Immunocore, Incyte, Merk Sharp \& Dome (MSD), Pfizer Oncology, Amgen, Astellas, Becton Dikinson (BD), Igea clinical Biophisics, Ivitech, Janssen oncology, Merck, Pierre Fabre Oncology, Assing, EuroClone, Fuidigm, PerkinElmer, Werfen, and under the auspices of the ACC, the AIOM, the SIC, the SIICA, the Associazione Italiana Oncologia Toracica (AIOT), the Fondazione Associazione Italiana per la Ricerca sul Cancro (AIRC), the Comune di Verona, the Università di Verona, the Federazione Nazionale degli Ordini dei Medici Chirurghi e degli Odontoiatri, the Fondazione Grazia Focacci, the Fondazione Italiana per la Ricerca sulle Malattie del 
Pancreas, the Fondazione Pezcoller, the Fondazione Melanoma Onlus, the Fondazione IRCCS Istituto Nazionale dei Tumori, the Istituto Oncologico Veneto (IOV), the Università di Verona, and the Women for Oncology Italy.

\section{Declarations}

Conflict of interest PFF has received honorarium for advisory board participation from: Bristol Meyers Squibb, Novartis, MSD, Pierre Fabre and Roche. MDN has received honorarium for advisory board participation from Bristol Meyers Squibb. VB reports relationship with IoBiotech Aps and Codiak BioScience (personal fees), outside the submitted work. All other authors have no conflict of interest to declare.

Ethical approval This article does not contain any studies with human participants or animals performed by any of the authors.

\section{References}

1. Petitprez F, de Reynies A, Keung EZ et al (2020) B cells are associated with survival and immunotherapy response in sarcoma. Nature 577:556-560. https://doi.org/10.1038/s41586-019-1906-8

2. Roumenina LT, Daugan MV, Noe R et al (2019) Tumor cells hijack macrophage-produced complement $\mathrm{C} 1 \mathrm{q}$ to promote tumor growth. Cancer Immunol Res 7:1091-1105. https://doi.org/10. 1158/2326-6066.CIR-18-0891

3. Lienart S, Merceron R, Vanderaa C et al (2018) Structural basis of latent TGF-beta1 presentation and activation by GARP on human regulatory $\mathrm{T}$ cells. Science 362:952-956. https://doi.org/10.1126/ science.aau2909

4. de Streel G, Bertrand C, Chalon N et al (2020) Selective inhibition of TGF-beta1 produced by GARP-expressing Tregs overcomes resistance to PD-1/PD-L1 blockade in cancer. Nat Commun 11:4545. https://doi.org/10.1038/s41467-020-17811-3

5. Kalafati L, Kourtzelis I, Schulte-Schrepping J et al (2020) Innate immune training of granulopoiesis promotes anti-tumor activity. Cell 183(771-85):e12. https://doi.org/10.1016/j.cell.2020.09.058

6. Calderaro J, Petitprez F, Becht E et al (2019) Intra-tumoral tertiary lymphoid structures are associated with a low risk of early recurrence of hepatocellular carcinoma. J Hepatol 70:58-65. https:// doi.org/10.1016/j.jhep.2018.09.003

7. Sautes-Fridman C, Petitprez F, Calderaro J, Fridman WH (2019) Tertiary lymphoid structures in the era of cancer immunotherapy. Nat Rev Cancer 19:307-325. https://doi.org/10.1038/ s41568-019-0144-6

8. Di Modugno F, Spada S, Palermo B et al (2018) hMENA isoforms impact NSCLC patient outcome through fibronectin/beta1 integrin axis. Oncogene 37:5605-5617. https://doi.org/10.1038/ s41388-018-0364-3

9. Rosenthal R, Cadieux EL, Salgado R et al (2019) Neoantigendirected immune escape in lung cancer evolution. Nature 567:479-485. https://doi.org/10.1038/s41586-019-1032-7

10. Eoli M, Corbetta C, Anghileri E et al (2019) Expansion of effector and memory $\mathrm{T}$ cells is associated with increased survival in recurrent glioblastomas treated with dendritic cell immunotherapy. Neurooncol Adv. 1:22. https://doi.org/10.1093/noajnl/vdz022

11. Norelli M, Camisa B, Barbiera G et al (2018) Monocyte-derived IL-1 and IL- 6 are differentially required for cytokine-release syndrome and neurotoxicity due to CAR T cells. Nat Med 24:739748. https://doi.org/10.1038/s41591-018-0036-4

12. Porcellini S, Asperti C, Corna S, Cicoria E, Valtolina V, Stornaiuolo A, Valentinis B, Bordignon C, Traversari C (2020) CAR
$\mathrm{T}$ cells redirected to CD44v6 control tumor growth in lung and ovary adenocarcinoma bearing mice. Front Immunol 11:99. https://doi.org/10.3389/fimmu.2020.00099

13. Sabatino M, Hu J, Sommariva M et al (2016) Generation of clinical-grade CD19-specific CAR-modified CD8+ memory stem cells for the treatment of human B-cell malignancies. Blood 128:519 528. https://doi.org/10.1182/blood-2015-11-683847

14. Montagner IM, Penna A, Fracasso G, Carpanese D, Dalla Pieta A, Barbieri V, Zuccolotto G, Rosato A (2020) Anti-PSMA CARengineered NK-92 cells: an off-the-shelf cell therapy for prostate cancer. Cells. https://doi.org/10.3390/cells9061382

15. Advani R, Flinn I, Popplewell L et al (2018) CD47 blockade by Hu5F9-G4 and rituximab in non-Hodgkin's lymphoma. N Engl J Med 379:1711-1721. https://doi.org/10.1056/NEJMoa1807315

16. Vanpouille-Box C, Alard A, Aryankalayil MJ et al (2017) DNA exonuclease Trex 1 regulates radiotherapy-induced tumour immunogenicity. Nat Commun 8:15618. https://doi.org/10.1038/ncomm s15618

17. Diamond JM, Vanpouille-Box C, Spada S et al (2018) Exosomes shuttle TREX1-sensitive IFN-stimulatory dsDNA from irradiated cancer cells to DCs. Cancer Immunol Res 6:910-920. https://doi. org/10.1158/2326-6066.CIR-17-0581

18. Formenti SC, Rudqvist NP, Golden E et al (2018) Radiotherapy induces responses of lung cancer to CTLA-4 blockade. Nat Med 24:1845-1851. https://doi.org/10.1038/s41591-018-0232-2

19. Gandhi L, Rodriguez-Abreu D, Gadgeel S et al (2018) Pembrolizumab plus chemotherapy in metastatic non-small-cell lung cancer. N Engl J Med 378:2078-2092. https://doi.org/10.1056/ NEJMoa1801005

20. Socinski MA, Jotte RM, Cappuzzo F et al (2018) Atezolizumab for first-line treatment of metastatic nonsquamous NSCLC. N Engl J Med 378:2288-2301. https://doi.org/10.1056/NEJMoa1716 948

21. Etxeberria I, Bolanos E, Quetglas JI et al (2019) Intratumor adoptive transfer of IL-12 mRNA transiently engineered antitumor CD8(+) T cells. Cancer Cell 36(613-29):e7. https://doi.org/10. 1016/j.ccell.2019.10.006

22. Perez-Ruiz E, Minute L, Otano I et al (2019) Prophylactic TNF blockade uncouples efficacy and toxicity in dual CTLA-4 and PD-1 immunotherapy. Nature 569:428-432. https://doi.org/10. 1038/s41586-019-1162-y

23. Schalper KA, Carleton M, Zhou M et al (2020) Elevated serum interleukin-8 is associated with enhanced intratumor neutrophils and reduced clinical benefit of immune-checkpoint inhibitors. Nat Med 26:688-692. https://doi.org/10.1038/s41591-020-0856-x

24. Kallikourdis M (2018) T cell responses to tumor: how dominant assumptions on immune activity led to a neglect of pathological functions, and how evolutionary considerations can help identify testable hypotheses for improving immunotherapy. Cancer Immunol Immunother 67:989-998. https://doi.org/10.1007/ s00262-017-2113-9

25. Bellone M, Brevi A, Huber S (2020) Microbiota-propelled T helper 17 cells in inflammatory diseases and cancer. Microbiol Mol Biol Rev. https://doi.org/10.1128/MMBR.00064-19

26. Sommariva M, Le Noci V, Bianchi F, Camelliti S, Balsari A, Tagliabue E, Sfondrini L (2020) The lung microbiota: role in maintaining pulmonary immune homeostasis and its implications in cancer development and therapy. Cell Mol Life Sci 77:2739-2749. https://doi.org/10.1007/s00018-020-03452-8

27. Blank CU, Rozeman EA, Fanchi LF et al (2018) Neoadjuvant versus adjuvant ipilimumab plus nivolumab in macroscopic stage III melanoma. Nat Med 24:1655-1661. https://doi.org/10.1038/ s41591-018-0198-0

28. Rozeman EA, Menzies AM, van Akkooi ACJ et al (2019) Identification of the optimal combination dosing schedule of neoadjuvant ipilimumab plus nivolumab in macroscopic stage III melanoma 
(OpACIN-neo): a multicentre, phase 2, randomised, controlled trial. Lancet Oncol 20:948-960. https://doi.org/10.1016/S14702045(19)30151-2

29. Versluis JM, Long GV, Blank CU (2020) Learning from clinical trials of neoadjuvant checkpoint blockade. Nat Med 26:475-484. https://doi.org/10.1038/s41591-020-0829-0

30. Ascierto PA, Ferrucci PF, Fisher R et al (2019) Dabrafenib, trametinib and pembrolizumab or placebo in BRAF-mutant melanoma. Nat Med 25:941-946. https://doi.org/10.1038/ s41591-019-0448-9

31. Minute L, Teijeira A, Sanchez-Paulete AR et al (2020) Cellular cytotoxicity is a form of immunogenic cell death. J Immunother Cancer. https://doi.org/10.1136/jitc-2019-000325

32. Majorini MT, Cancila V, Rigoni A et al (2020) Infiltrating mast cell-mediated stimulation of estrogen receptor activity in breast cancer cells promotes the luminal phenotype. Cancer Res 80:2311-2324. https://doi.org/10.1158/0008-5472.CAN-19-3596

33. Truffi M, Colombo M, Sorrentino L et al (2018) Multivalent exposure of trastuzumab on iron oxide nanoparticles improves antitumor potential and reduces resistance in HER2-positive breast cancer cells. Sci Rep 8:6563. https://doi.org/10.1038/ s41598-018-24968-x

34. Mosele F, Remon J, Mateo J et al (2020) Recommendations for the use of next-generation sequencing (NGS) for patients with metastatic cancers: a report from the ESMO Precision Medicine Working Group. Ann Oncol. https://doi.org/10.1016/j.annonc. 2020.07.014

35. Protti MP, De Monte L (2020) Dual role of inflammasome adaptor ASC in cancer. Front Cell Dev Biol 8:40. https://doi.org/10.3389/ fcell.2020.00040
36. Brunetto E, De Monte L, Balzano G et al (2019) The IL-1/IL-1 receptor axis and tumor cell released inflammasome adaptor ASC are key regulators of TSLP secretion by cancer associated fibroblasts in pancreatic cancer. J Immunother Cancer 7:45. https://doi. org/10.1186/s40425-019-0521-4

37. Calcinotto A, Spataro C, Zagato E et al (2018) IL-23 secreted by myeloid cells drives castration-resistant prostate cancer. Nature 559:363-369. https://doi.org/10.1038/s41586-018-0266-0

38. Bulgarelli J, Tazzari M, Granato AM et al (2019) Dendritic cell vaccination in metastatic melanoma turns "non-T cell inflamed" into "T-cell inflamed" tumors. Front Immunol 10:2353. https:// doi.org/10.3389/fimmu.2019.02353

39. Matet A, Ait Rais K, Malaise D et al (2019) Comparative cytogenetic abnormalities in paired choroidal melanoma samples obtained before and after proton beam irradiation by transscleral fine-needle aspiration biopsy and endoresection. Cancers (Basel). https://doi.org/10.3390/cancers 11081173

40. Damato BE, Dukes J, Goodall H, Carvajal RD (2019) Tebentafusp: T cell redirection for the treatment of metastatic uveal melanoma. Cancers (Basel). https://doi.org/10.3390/cancers110 70971

Publisher's Note Springer Nature remains neutral with regard to jurisdictional claims in published maps and institutional affiliations. 